\title{
Spectral linewidth and tuning requirements of sources for gas sensing in space-based applications at $935 \mathrm{~nm}$
}

\author{
D. Mclnerney, M. Lynch, J.F. Donegan and V. Weldon
}

\begin{abstract}
The spectral linewidth and tuning requirements of an external cavity laser (ECL) in the Littrow architecture and a distributed feedback (DFB) device for space-based gas sensing were investigated. The wavelengths of both devices were in the $935 \mathrm{~nm}$ range and different linewidth measurment techniques were explored for the two sources. The linewidth of the ECL was measured using a short-arm homodyne technique, while the linewidth of the DFB was measured with a heterodyne technique using the ECL as a local oscillator. The spectral linewidth of the ECL was measured to be $<300 \mathrm{kHz}$ with $<5 \mathrm{MHz}$ for the DFB device. The emission wavelength tuning characteristics, including the overall tuning range and the free running stability of the ECL, were also examined. The full frequency drift of the ECL measured over a 36-hour period was found to be approximately $540 \mathrm{MHz}$, while it showed approximately $0.4 \mathrm{~nm}$ continuous mode-hop free wavelength tuning.
\end{abstract}

\section{Introduction}

The objective of this work is the stabilisation of four laser frequencies to four specific predefined wavelengths that are associated with water absorption lines in the 935$940 \mathrm{~nm}$ range for space-based gas sensing. A list of these water absorption lines, with their wavelengths and linestrengths, is shown in Table 1 . The experimental set-up involves locking one injection seed laser (ISL) to the strongest water line via a water vapour reference gas cell. This laser is then used as a reference to stabilise the other lasers, each to one of the three remaining lines. Locking by means of gas absorption is the preferred technique as the absorption wavelength at a fixed temperature and pressure is constant. The stabilisation technique used for the three remaining lasers employs a Fabry-Perot interferometer to reference from the locked ISL by a fixed detuning. The work performed in this research is a portion of a wider study being carried out by the European Space Agency (ESA). The overall project is termed the WALES [water vapour light detection and ranging (LIDAR) experiment in space] mission and its objective is to provide better insight into water vapour, temperature and aerosol distribution in the upper troposphere and lower atmosphere. Sampling both strong and weak water vapour absorption lines at different wavelengths in the $935 \mathrm{~nm}$ region can achieve a complete water vapour absorption profile across an entire altitude range. Further information on the WALES mission can be found in [1].

The required optical characteristics of the ISL specified for this project include, an output power $>10 \mathrm{~mW}$,

\footnotetext{
(C) IEE, 2005

IEE Proceedings online no. 20050054

doi:10.1049/ip-opt:20050054

Paper first received 2nd June and in revised form 10th September 2005

The authors are with the Semiconductor Photonics Group, Physics Department, Trinity College, Dublin 2, Ireland

E-mail: dmcinern@tcd.ie
}

linewidth $<1 \mathrm{MHz}$, continuous tuning range $<0.5 \mathrm{~nm}$, frequency stability $<50 \mathrm{MHz}$ and free-running frequency drift $<500 \mathrm{MHz}$. Many devices were initially considered as a possible candidate for the ISL but because of the specified wavelength in the $935 \mathrm{~nm}$ region, an external cavity laser (ECL) was chosen as the preferred laser technology for the ISL, as it is capable of meeting all the necessary optical requirements. It has long been recognised that semiconductor lasers in an external cavity architecture have extremely narrow spectral linewidths, $<1 \mathrm{MHz}$. In some particular external cavity arrangements, linewidths of the order of kilohertz have been achieved [2-5]. The ECL used here is in the Littrow configuration and was supplied by Sacher Lasertechnik [6]. However, ECL's are not environmentally robust and would be therefore unsuitable for space-based operation because of the mechanical design of the device but nevertheless are used here as a proof of principle approach. A distributed feedback (DFB) laser was also investigated as a possible alternative ISL. It should be noted that the preferred technology is a DFB laser but at the commencement of this work, these lasers were unable to meet all of the optical requirements specified, so the ECL option provides the best substitute. One of the biggest problems in realising high quality single frequency lasers in the traditional and best understood material systems (GaAs/AlGaAs) for short wavelength lasers $(<1 \mu \mathrm{m})$ is the oxidation of the Al of the AlGaAs overgrown layers during the grating writing process. This impairment has traditionally reduced the grating quality and the resultant control over the coupling ratio and the device. The coupling ratio is defined as the ratio of the optical power in the grating region to the total optical power of the DFB and is dependent on the quality of the grating. Recent results on new means of creating metal gratings on the sides of the ridge waveguide, thus inducing complex coupling in the cavity, have shown good single mode yields and side mode suppression. This technique does not require the overgrown step required to date for the most widely used material system in this wavelength 
Table 1: Water absorption lines and corresponding line-strengths

\begin{tabular}{lllr}
\hline $\begin{array}{l}\text { Water absorption } \\
\text { lines }\end{array}$ & $\begin{array}{l}\text { Wavelength } \\
\text { air }(\mathrm{nm})\end{array}$ & $\begin{array}{l}\text { Wavelength } \\
\text { vacuum }(\mathrm{nm})\end{array}$ & $\begin{array}{r}\text { Line-strengths } \\
\text { (cm/molecule) }\end{array}$ \\
\hline Weak line & 935.657 & 935.9065 & $4.15 \times 10^{-24}$ \\
Medium strength line & 935.307 & 935.56116 & $5.4 \times 10^{-23}$ \\
Strong line & 935.427 & 935.68459 & $6.45 \times 10^{-22}$ \\
Off line & 935.6 & 935.8564 & $6 \times 10^{-26}$ \\
\hline
\end{tabular}

range. Other Al-free material systems are available in the wavelength range but are still at an early stage for realising single longitudinal mode laser sources [7].

The DFB is the preferred device for the ISL because it is a much more robust laser than the ECL. Also, care was taken in the ISL design to ensure that the ECL can be replaced by a DFB laser with only minimal changes. It is envisaged that substitution of the ECL with a fibrecoupled DFB can be easily achieved because of the reduced dimensions of the fibre-coupled DFB laser source at $935 \mathrm{~nm}$. The main alterations required for DFB substitution involve changes to the relevant settings for the temperature controller and the gain current and the different connections needed for the DFB. The overall architecture would be left unaffected by the substitution of the laser source. The DFB was supplied by Nanoplus. Both devices had a room temperature emission wavelength at $935 \mathrm{~nm}$ and side mode suppression ratio (SMSR) $>30 \mathrm{~dB}$. A frequency modulation spectroscopy (FMS) technique is used when locking the emission wavelength of an ECL to the strongest absorbing water line. A detailed description of FMS is given in [8-10]. Once the laser emission is passed through a reference cell, the demodulated signal of the incident light is recorded and consists of pure absorption and pure dispersion components. The photodetector signal can then be resolved into in-phase and quadrature components, which can be measured separately using phase sensitive detection. There exist many methods for frequency stabilisation of diode lasers but in this case it is achieved by locking to the zero crossing point of the quadrature component of the demodulated signal, which corresponds to the centre of the water absorption line [11-15]. Monitoring the recorded quadrature signal and adjusting the gain current and piezo values of the ECL to compensate for any drifts from the line centre, maintains the desired locking frequency. Achieving an extremely stable frequency reference by means of locking to a gas absorption line is a very powerful tool. However, in order to maximise the potential of the method, an investigation of certain emission characteristics of the laser devices involved is needed. For example, the accuracy of the frequency locking of the laser is partly limited by the resolution of the spectroscopic detection method. As a result, the spectral linewidth of an ECL and a DFB and the emission wavelength tuning capabilities of the ECL at $935 \mathrm{~nm}$ have been examined. The free running emission frequency stability of the ECL is also examined using a suitable gas absorption line. The temperature and pressure of gas in the cell are held constant to ensure spectral and amplitude stability of the absorption line. The technique involves setting the laser wavelength to the side of the gas absorption line and monitoring the transmitted power. The laser emission is directed through the gas cell and the laser frequency variations are converted to amplitude variations in the absorption/ transmitted signal.

\section{Laser spectral linewidth theory}

The fundamental spectral emission linewidth of a laser is associated with fluctuations in the phase of the optical field. These fluctuations arise from carrier density oscillation and spontaneous emission events of the gain medium into the cavity mode. In the optical field emitted from a laser, a stimulated emission event adds a photon to the field and the added photon has the same phase as the field already in the cavity. A spontaneous emission adds a photon whose phase is different to the phase of the field in the cavity. As a result of the addition of a spontaneous emission photon, the phase of the optical field obtains a kick and over time, induces a phase change to the field. In addition to the spontaneous emission event, the amplitude of the optical field is also changed. By means of relaxation oscillations, the field decays to its steady state value but this causes an additional phase change. The relaxation oscillations that occur after a spontaneous event involve carrier density fluctuations that induce refractive index changes causing additional phase noise and a broader linewidth, unique to semiconductor lasers. As a result, the output of the laser is not perfectly monochromatic but exhibits some degree of phase noise defined by the spectral linewidth. The spectral linewidth of lasers is also extremely important in the field of coherent optical communication systems as well as spectroscopic measurements and therefore has been studied in great detail by many authors [16-18].

\section{Experimental}

Many methods exist for the measurement of the linewidth of laser devices depending on the particular device parameters. The wavelengths of the ECL and DFB devices under investigation here are in the $935 \mathrm{~nm}$ range and so a delayed selfhomodyne interferometric technique using kilometres of single mode optical fibre at $935 \mathrm{~nm}$ was considered prohibitively expensive. Hence, a short-arm homodyne technique was employed for measurement of the ECL linewidth. The short-arm method is favoured because of the long coherence length of the ECL. The linewidth of the DFB was also measured with a heterodyne technique using the ECL as a local oscillator (LO).

In the former arrangement, the emitted optical field of the ECL is mixed with a delayed version of itself. ECL's have very narrow linewidths because of their unique architecture, which employs quite a long external cavity. The ECL used in these measurements had an external cavity length of approximately $3 \mathrm{~cm}$. The effect of this external cavity reduces the linewidth, so the delay in the short-arm set-up is fixed to be shorter than the coherence length of the ECL under test. Therefore the two arms of the ECL optical field mix coherently and a number of interference peaks appear in the detected power spectrum. The free 
spectral range of the peaks should correspond to the length of fibre used. The output of the ECL is split into two equal parts by means of a $50 / 50$ coupler. One arm of the coupler is directed to $30 \mathrm{~m}$ of fibre, which is much shorter than the coherence length of the laser. The other beam is passed through a polarisation controlling stage. The polarisation controller consists of just three loops of fibre, which can be mechanically rotated. The two beams are then recombined with another 50/50 coupler and detected in a highspeed detector. When the two coherent fields are combined at the detector, a difference frequency signal is generated which contains the combined FM field of the arm delayed by the $30 \mathrm{~m}$ of fibre and the arm that passes through the polarisation controller. Essentially, the technique converts the combined optical power spectrum to an electrical spectrum, which can be easily measured using an RF spectrum analyser. However, because this electrical spectrum contains the noise of the two combined signals, the $-3 \mathrm{~dB}$ FWHM centred at $0 \mathrm{~Hz}$ is equal to twice the actual linewidth of the laser under test.

The experimental set-up for the self-homodyne technique used for the measurement of the spectral linewidth of the ECL is shown in Fig. 1. The ECL has a maximum output power $>10 \mathrm{~mW}$ at room temperature and emission wavelength at $935 \mathrm{~nm}$. The output of the ECL is fibre-coupled via an integral collimator and a single mode fibre but unfortunately there was no optical isolator included. Therefore the linewidth measurements, in the absence of an isolator, proved quite difficult since the short-arm measurement profile (Lorentzian envelope function with an underlying interference pattern) was very susceptible to masking by the effects of feedback to the laser resulting from stray reflections from fibre ends or by spurious optical cavities. In practice, the profile was non-ideal. In spite of careful set-up and repeated optimisation of the measurement system, the linewidth measurement results were considered unreliable attributed to feedback from the fibre coupling stage. Hence, it was decided to adopt a different approach and the measurements were repeated under different conditions. The emission from the ECL was directly coupled out through free space, bypassing the collimator and fibre. The linewidth measurements were then repeated. In this arrangement, the free-space emission was directed to an off-axis parabolic (OAP) mirror to eliminate feedback from refractive optical elements, before being coupled via an angle-polished FC-APC fibre in to the Mach-Zehnder fibre interferometer. The geometry of this optical set-up was such that the feedback to the ECL was attenuated and

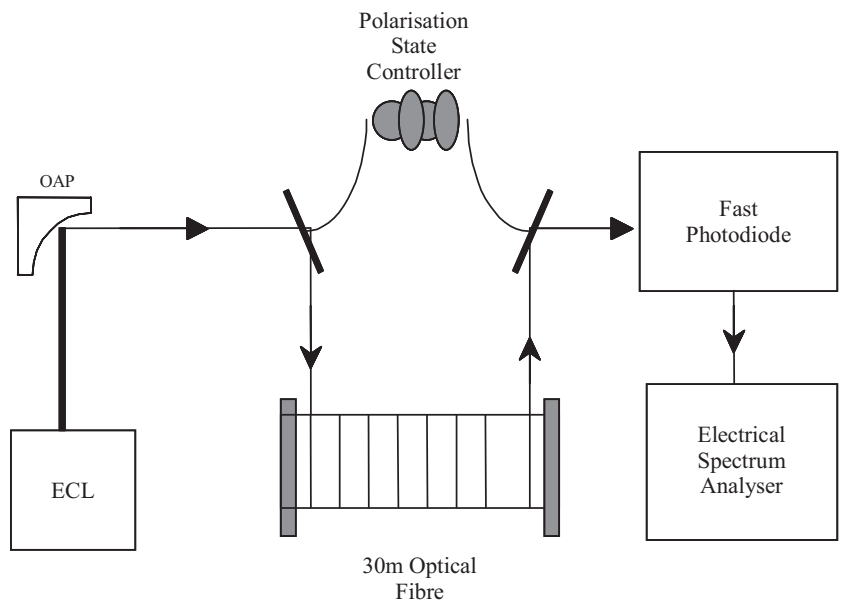

Fig. 1 Experimental set-up for ECL emission linewidth measurement its effects mitigated. Essentially, non-optimum coupling to the interferometer with $10 \mathrm{~dB}$ loss provided an effective $20 \mathrm{~dB}$ isolation and decreased the sensitivity to back reflections from the first coupling stage. The detrimental effects of unwanted feedback were clearly present for the initial linewidth measurements but were also observed when recording power plane measurements of the ECL. The gain current and piezo voltage are scanned with the output power of the ECL recorded. The effect of the feedback is easily observed in the power plane shown in Fig. $2 a$ in contrast to the plane in Fig. $2 b$ where unwanted feedback is not present. Fig. $2 a$ show the effects of the feedback, which clearly removes the option of continuous wavelength tuning of the device. The overall performance of the ECL could be improved considerably if an internal optical isolator, before fibre-optic coupling, is installed. Optical isolators at $935 \mathrm{~nm}$ are bulky and expensive and would be difficult to implement in an overall ECL packaged device but are critical for optimum performance.

The spectral linewidth of the DFB laser was also measured, with a heterodyne technique using the ECL as a LO, since its linewidth was expected to be significantly less that that of the DFB device. The set-up for the heterodyne technique is shown in Fig. 3. In this experiment, the ECL output was directly fibre-coupled to a free space collimator. The feedback effects to the ECL were again attenuated and reduced allowing a more accurate evaluation of the spectral linewidth of the DFB. For this measurement technique, the emission wavelength of the ECL is tuned to a frequency close to that of the laser under test and is fixed during the experiment. This creates a heterodyne beat tone between the LO and each of the frequency components in the signal spectrum as shown in Fig. 4. From Fig. 5, it is clear to see that for this measurement, the emission wavelength of the ECL was tuned to within approximately $4 \mathrm{GHz}$ of the frequency of DFB.

This was achieved with careful adjustment of the ECL operating parameters and using a suitable bandwidth photodetector, while monitoring the ECL emission wavelength with a wavelength meter. A summary of the theory behind the technique is given below and a more detailed description can be found in [19]. Consider the two optical fields of both LO and DFB devices incident on the photodetector, after passing through a 50/50 coupler

$$
\begin{aligned}
E_{\mathrm{s}}(t) & =\sqrt{P_{\mathrm{s}}(t)} e^{j\left(2 \pi v_{\mathrm{s}} t+\phi_{\mathrm{s}}(t)\right)} \\
E_{\mathrm{LO}}(t) & =\sqrt{P_{\mathrm{LO}}(t)} e^{j\left(2 \pi v_{\mathrm{LO}} t+\phi_{\mathrm{LO}}(t)\right)}
\end{aligned}
$$

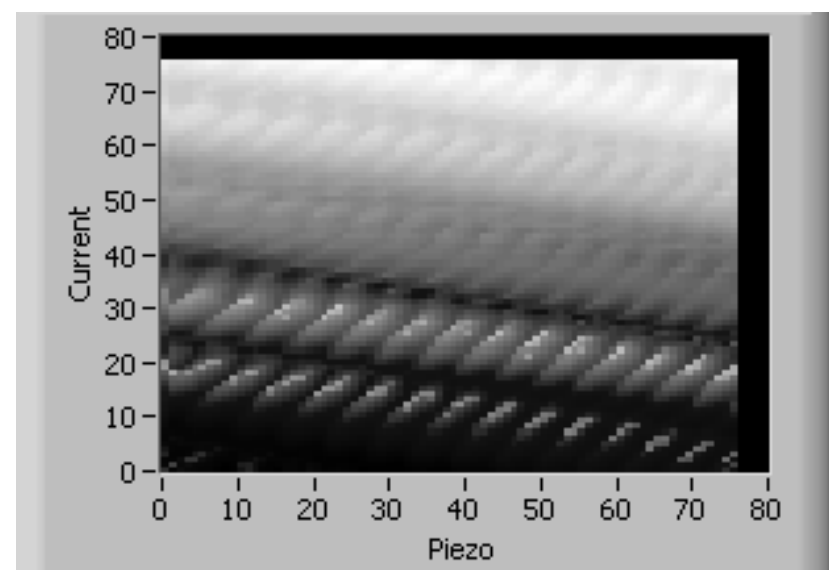

Fig. 2a Unwanted feedback effects present in power plane of ECL 


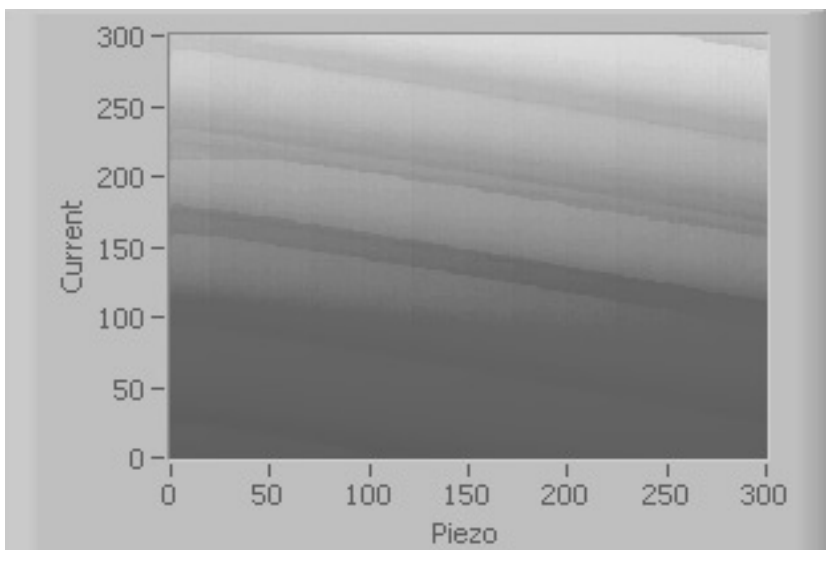

Fig. $2 \boldsymbol{b}$ Power plane of ECL without any external feedback effects

The total field at the photodetector is thus given by (3)

$$
E_{\mathrm{T}}(t)=E_{\mathrm{s}}(t)+E_{\mathrm{LO}}(t)
$$

Since we are detecting the amplitude and not the optical field

$$
P(t)=\left|E_{\mathrm{T}}(t)\right|^{2}
$$

the quadratic response allows the detection of the interference between the two fields. The photocurrent generated in the detector is thus given by (5)

$$
i(t)=R\left|E_{\mathrm{T}}(t)\right|^{2}
$$

with the responsivity of the detector given by $R$.

By substitution we obtain (6)

$$
\begin{aligned}
i(t)= & R\left[P_{\mathrm{s}}(t)+P_{\mathrm{LO}}+2 \sqrt{P_{\mathrm{s}}(t) P_{\mathrm{LO}}} \cos \left(2 \pi\left(f_{1 \mathrm{~F}}\right) t\right.\right. \\
& +\Delta \phi(t))]
\end{aligned}
$$

The first two terms correspond to the direct intensity detection, while the third term is the important heterodyne mixing term. Note, the dependence on the actual optical frequency is gone and only the difference remains $\left(f_{\mathrm{IF}}\right)$. It is important to note that if either field were separately detected on the photodiode, the resulting photocurrent would follow only the power variations $P(t)$ and all phase information would be lost.

A polarisation state controller is placed in the path of the ECL (LO) in order to align the polarisation of the LO to that of the signal under test. Matching the polarisation of the two signals maximises the signal strength of the detected electrical field. The key requirement of this technique is to have a reference laser with a stable narrow spectral linewidth. The reason for this is that the interference of the two optical fields is what is actually measured and so it is assumed

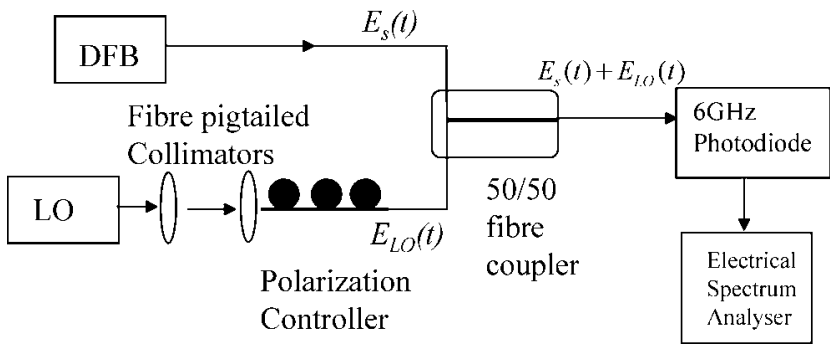

Fig. 3 Experimental set-up for DFB emission linewidth measurement

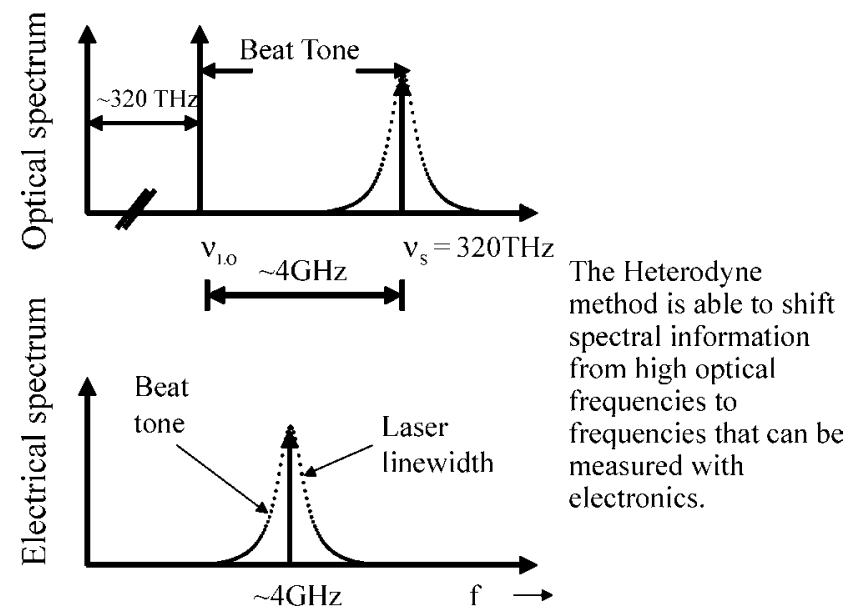

Fig. 4 Heterodyne beat tone between the $L O$ and DFB

that the linewidth of the LO is much smaller with respect to the laser under test. If this is the case, then the beat tone will be broadened primarily by the phase noise of the laser under study. It is also beneficial to have a wide bandwidth detector. A $6 \mathrm{GHz}$ photoreceiver was used in the experiment allowing the tuning of the LO frequency to the DFB to be easily observed.

A wavelength meter was also used, instead of an OSA, to add extra accuracy when matching the wavelength of the DFB under test and the ECL. The sensitivity of the technique can be improved by increasing the output power of the LO. This is explained by the fact that the strength of the detected power spectrum increases with LO power, $P_{\mathrm{LO}}$. The electrical spectrum of the detected signal contains information on the direct detection terms and products of optical mixing terms.

$$
S_{i}(f) \simeq R^{2}\left\{S_{\mathrm{d}}(f)+2\left[S_{\mathrm{LO}}(v) \otimes S_{\mathrm{s}}(-v)\right]\right\}
$$

The first term is just the direct detection term, while the second term is the one of interest. It is the heterodyne mixing product that is the convolution of the LO spectrum $S_{\mathrm{LO}}(v)$ with the signal spectrum $S_{\mathrm{s}}(v)$. The convolution originates from the multiplication of the time varying oscillator field with the signal field in the photodetector. Multiplication in the time-domain is equivalent to convolution in the frequency domain. Therefore the detected

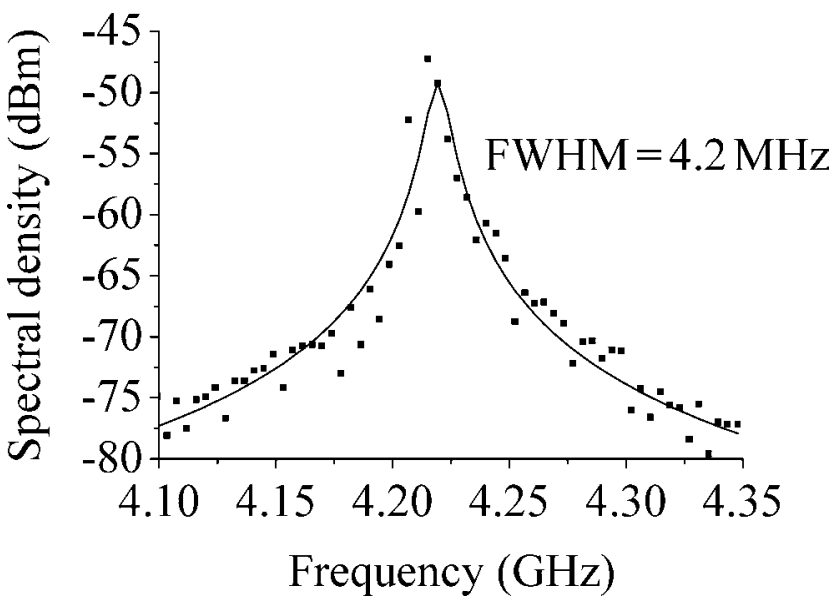

Fig. 5 DFB linewidth measurement with measured and Lorentzian fitted data giving a result of $4.2 \mathrm{MHz}$ 
electrical spectrum will be given by (8),

$$
S_{i}(f) \simeq 2 R^{2} P_{\mathrm{LO}} S_{\mathrm{s}}\left(v-v_{\mathrm{LO}}\right)
$$

\section{Emission linewidth results}

The resulting electrical spectrum of the linewidth measurement on the ECL by the short-arm homodyne method is displayed in Fig. 6. The resulting linewidth shape was fitted and a result of approximately $230 \mathrm{kHz}$ was obtained for the spectral linewidth of the ECL. With the short-arm method the optical fields are mixing coherently and so the interference peaks are quite evident in the trace of the electrical spectrum. The spectral linewidth of the DFB was measured with a heterodyne technique using the ECL as a LO. The ECL output was directly fibre-coupled to a free space collimator. The FWHM and therefore the linewidth of the DFB, was calculated to be approximately $4.2 \mathrm{MHz}$ as shown in Fig. 5. This result is consistent with the values specified by Nanoplus which state that the linewidth of their $935 \mathrm{~nm}$ DFB lasers typically lie below $5 \mathrm{MHz}$.

\section{$5 \quad$ Free running stability}

The free running emission frequency stability of a laser can be measured using a suitable water vapour absorption line as an absolute wavelength reference. The temperature and pressure of the water vapour in a gas cell are held constant to ensure spectral and amplitude stability of the absorption line. The technique involves setting the laser wavelength to the side of a water absorption line and monitoring the transmitted power. The laser emission is directed through a gas cell and laser frequency variations are converted to amplitude variations in absorption/transmitted signal. These frequency variations converted to amplitude variations are indicated in Fig. 7. Measurements are made using a $14 \mathrm{~s}$ test bandwidth. The results are shown in Fig. 8. The time range corresponds to approximately $36 \mathrm{~h}$. The ECL was continuously operated without adjustment for the duration of the test. The full frequency drift measured over the 36hour period is approximately $540 \mathrm{MHz}$. The ECL source can operate as a very precise water vapour sensor in space provided the frequency locking scheme is maintained during operation. If the locking is turned off or fails, the

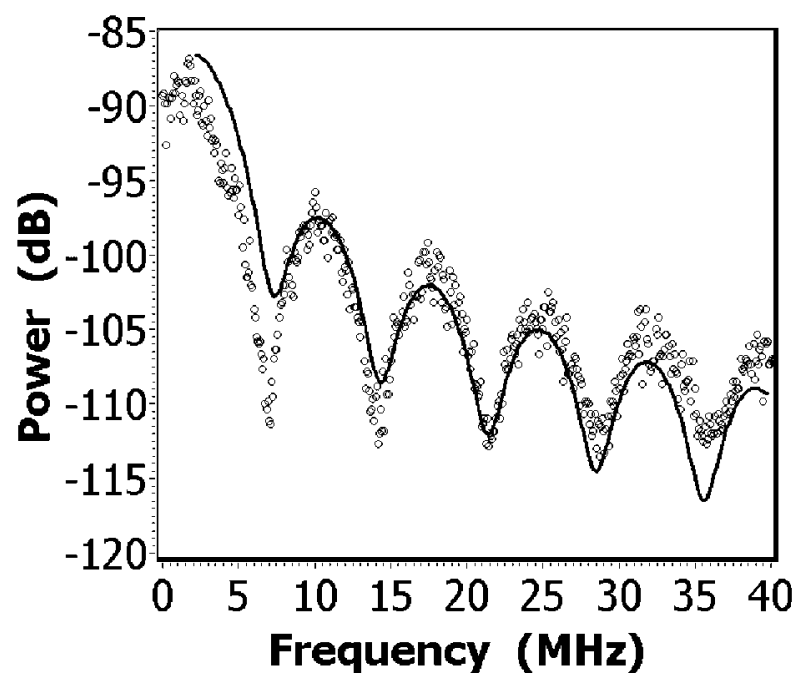

Fig. 6 Trace above shows the measured and fitted $R F$ power (in $d B \mathrm{~m}$ ) in the range $0-40 \mathrm{MHz}$ giving a value of $230 \mathrm{kHz}$ for the linewidth of the ECL

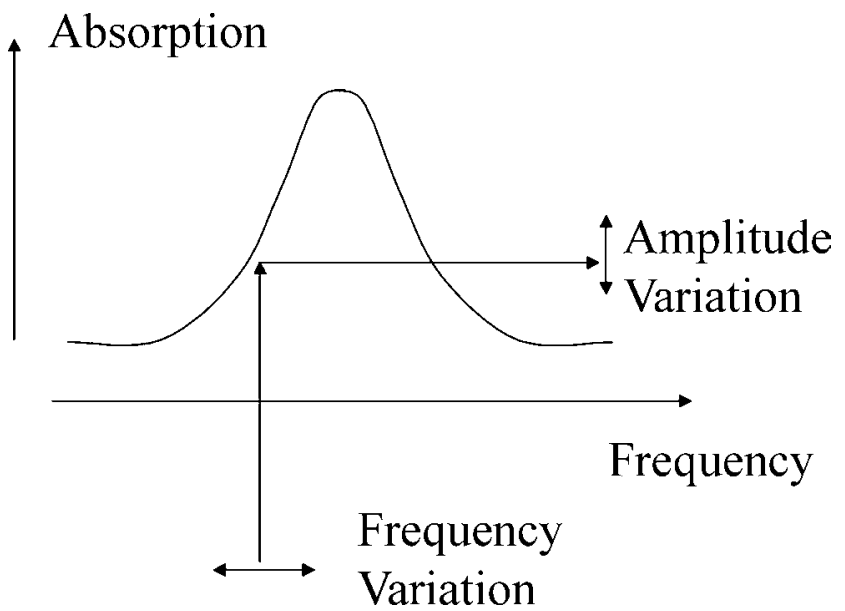

Fig. 7 Conversion of frequency variations to amplitude variations on side of strong gas absorption line, with temperature and pressure kept constant, which was used to measure frequency drift of ECL

relatively large frequency drift of the ECL may have a detrimental effect on the detection system. Consider, if the locking is switched back on and the emission frequency of the ECL has drifted away from the peak of the gas absorption line. Locking will only be re-established if the drift is such that the emission frequency is within the central linear sloping portion of the if demodulated harmonic signal, which is equivalent to somewhat less than the half width of the gas absorption linewidth. However, since the measured frequency drift of $>500 \mathrm{MHz}$ occurs over a long time scale (hours), locking will be reestablished provided the down time is relatively short (less than several hours). Another factor here is of course the absorption linewidth of the gas in the reference cell used to achieve locking. Hence, careful specification of the pressure of the gas in the cell is important.

\section{Wavelength tuning characteristics of ECL}

The overall tuning capabilities of the ECL were also examined in detail. The piezo voltage for tuning the ECL grating can be controlled over a $0-150 \mathrm{~V}$ range, so a complete wavelength tuning profile can be measured. An example of a power contour plane is shown in Fig. 9. For this particular power plane, the gain current is scanned from $80 \mathrm{~mA}$ to

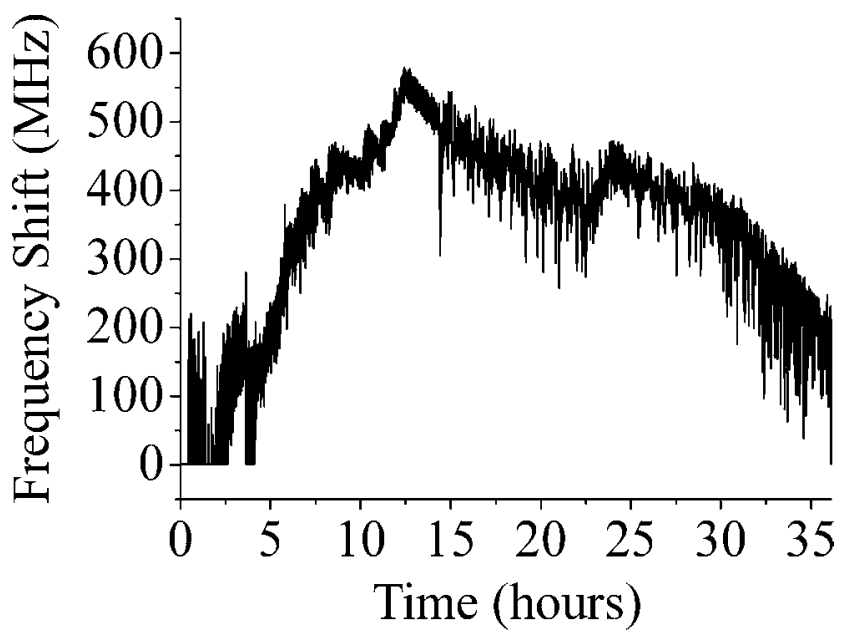

Fig. 8 Frequency shift of ECL against time over approximately $36 \mathrm{~h}$ 


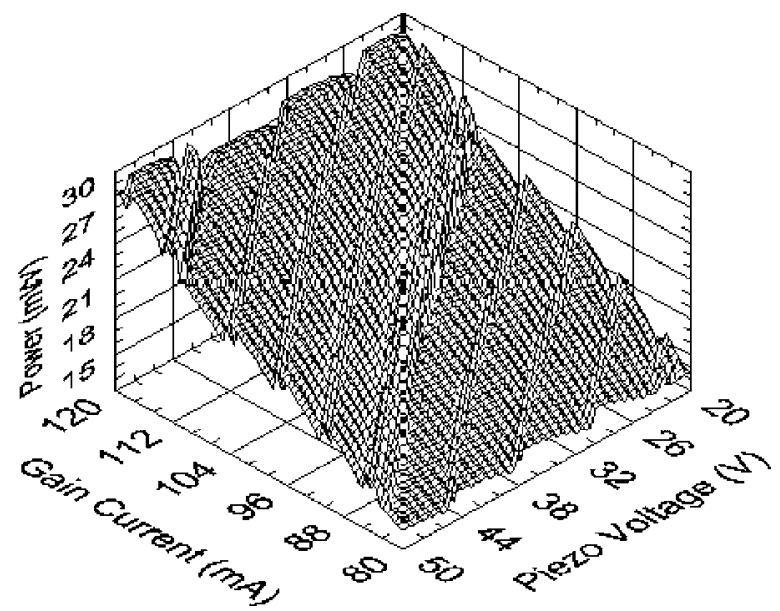

Fig. 9 Contour plot of power plane for gain current and piezo tuning of ECL, with dashed line showing continuous wavelength tuning over a super-mode

approximately $120 \mathrm{~mA}$ and the piezo voltage from 20 to $50 \mathrm{~V}$. The power plane is simply recorded by the photodiode on the back end of the device chip, as the gain current and piezo voltage are scanned. Essentially, the gain current is set to certain values above threshold and the piezo is swept across a range from 0 to $150 \mathrm{~V}$, depending on how much tuning is required.

Continuous tuning can then be achieved by tuning both piezo and gain current simultaneously along one of the so-called super modes, as indicated by the dashed white line in the graph. However, it should be noted that while tuning piezo and gain current simultaneously, it is extremely important to allow a time delay after each stepped change in gain current to allow the ECL to temperature stabilise in order to provide more accurate tuning capabilities. The time response for this thermal stabilisation of the ECL was also investigated. This was achieved by tuning the emission wavelength of the ECL to the minimum of the quadrature demodulated signal of the strong absorption line at $935.684 \mathrm{~nm}$. The emission wavelength is then abruptly tuned, from point $\mathrm{A}$ to point $\mathrm{B}$, to the maximum of the quadrature demodulated signal of the same strongly absorbing water vapour line at $935.684 \mathrm{~nm}$ as indicated in Fig. 10. The received photodiode amplitude and the received quadrature response are then recorded for the abrupt change in wavelength. The thermal stabilisation

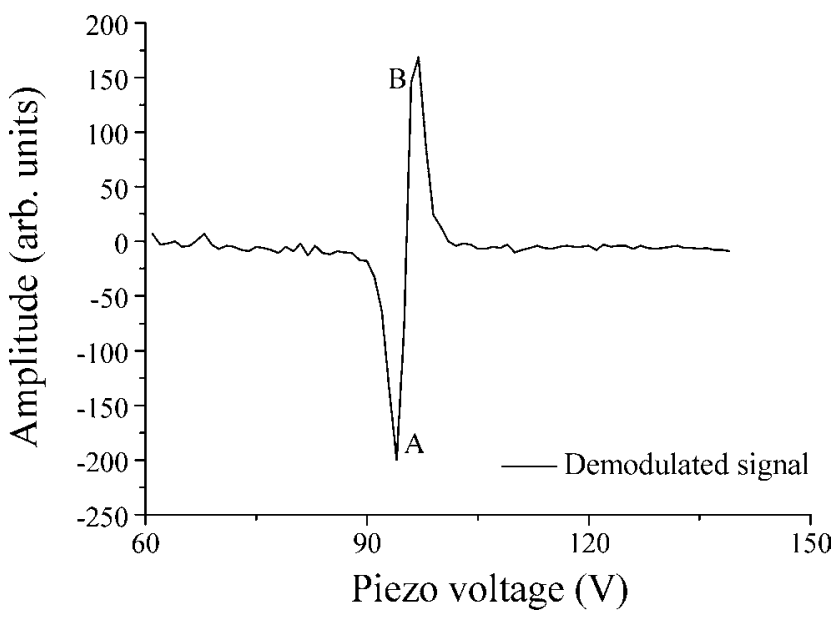

Fig. 10 Demodulated signal of the strongly absorbing water vapour line at $935.684 \mathrm{~nm}$ with points $A$ and $B$ indicated for thermal stabilisation test

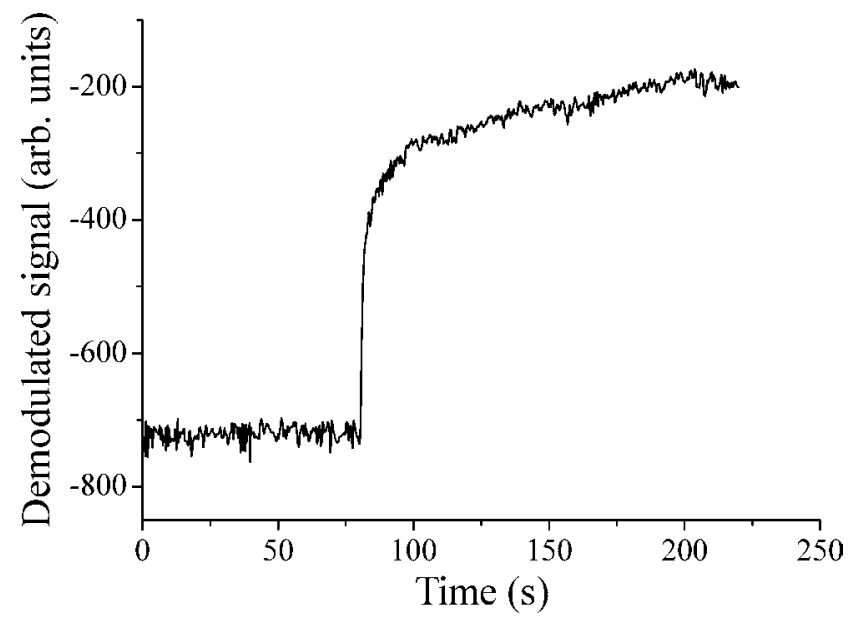

Fig. 11 Recorded quadrature demodulated signal for change in piezo voltage from point A to point B (from Fig. 10), for thermal stabilisation time corresponding to approximately $2.4 \mathrm{~min}$

time response of the ECL is then calculated by monitoring the time for the received power and quadrature demodulated signal to stabilise as shown in Fig. 11. The time recorded for the demodulated signal to stabilise was approximately 2.4 min but the temperature controller of the ECL also affects this result. As stated earlier, this particular ECL did not have an optical isolator included in the set-up and so was susceptible to unwanted feedback effects. These feedback effects to the laser caused by stray reflections from fibre ends or by other spurious optical cavities, have quite a detrimental effect of the ECL performance. As a result, every precaution to minimise feedback to the ECL was taken. It was found that by defocusing the collimating objective on the ECL, although this reduced the output power, actually improved the overall performance of the ECL by helping somewhat to reduce the feedback. By exploiting the full piezo and gain current tuning range, approximately $0.4 \mathrm{~nm}$ continuous, mode-hop free, wavelength tuning is possible with the ECL. This range is sufficient to cover the four specific, predefined wavelengths that are associated with water absorption lines previously mentioned.

\section{Conclusion}

The emission linewidth of an ECL in the Littrow configuration and a DFB device, both with wavelengths at $935 \mathrm{~nm}$, were measured using a short-arm homodyne technique and a heterodyne technique using the ECL as an LO, respectively. The experimental set-up for these techniques was adapted in order to overcome the problem with unwanted external optical feedback to the devices. Stray reflections from either fibre ends or by spurious optical cavities within the device architecture were the main sources of feedback. The detrimental effect of the feedback was aggravated by the fact that neither device was fitted with an optical isolator. However, it was found that by arranging a free space experimental set-up, the effects of the feedback could be reduced. As a result, successful measurements yielded spectral linewidth values of $<300 \mathrm{kHz}$ for the ECL and $<5 \mathrm{MHz}$ for the DFB device. The full frequency drift of the ECL measured over a 36-hour period was found to be approximately $540 \mathrm{MHz}$. The wavelength tuning capabilities of the ECL was also investigated. It was found that $0.4 \mathrm{~nm}$ continuous mode-hop free wavelength tuning is possible using the combined total tuning range of the gain current and piezo voltage. The thermal time 
response of the ECL was also measured to be approximately $2.4 \mathrm{~min}$, which clearly shows the limited thermal capabilities of the ECL. This disadvantage of the ECL is especially evident for fast emission wavelength tuning and accurate recording of the power planes.

\section{References}

1 http://www.esa.int/esaLP/ESA40MPV16D_earthexplorers_0.html

2 Wyatt, R.: 'Spectral linewidth of external cavity semiconductor lasers with strong frequency-selective feedback', Electron. Lett., 1985, 21, pp. $658-659$

3 Wyatt, R., and Devlin, W.J.: ' $10 \mathrm{kHz}$ linewidth $1.5 \mu \mathrm{m}$ InGaAsP external cavity laser with $55 \mathrm{~nm}$ tuning range', Electron. Lett., 1984, 19, pp. 110-112

4 Favre, F., le Guen, D., Simon, J.C., and Landousies, B.: 'Externalcavity semiconductor laser with $15 \mathrm{~nm}$ continuous tuning range', Electron. Lett., 1986, 22, pp. 795-796

5 Agrawal, G.P.: 'Line narrowing in a single mode injection laser due to external optical feedback', IEEE J. Quantum Electron., 1984, QE-20, pp. $468-471$

6 http://www.sacher-laser.com

7 Fischer, M., Gollub, D., and Reinhardt, M.: 'Advances in GaInNAs edge emitting laser diodes'. Proc. Int. 8th Microptics Conference (MOC '01), Osaka, Japan, October 24-26, 2001 (invited)

8 Silver, J.A.: 'Frequency modulation spectroscopy for trace species detection: theory and comparison among experimental methods', Appl. Opt., 1992, 31, pp. 707-717
9 Measures, R.M.: 'Laser remote chemical analysis' (John Wiley \& Sons, 1988), p. 236

10 Bomse, D.S., Stanton, A.C., and Silver, J.: 'Frequency modulation and wavelength modulation spectroscopies: comparison of experimental methods using a lead salt diode laser', Appl. Opt., 1992, 31, pp. $717-731$

11 Tsuchida, H., Ohtsu, M., and Kato, T.: 'Frequency stabilization of $\mathrm{AlGaAs}$ semiconductor laser to the absorption line water vapor', Jpn. J. Appl. Phys., 1982, 21, LI-L3

12 Ohtsu, M., Kotani, H., and Tagawa, H.: 'Spectral measurements of $\mathrm{NH}_{3}$ and $\mathrm{H}_{2} \mathrm{O}$ for pollutant gas monitoring by $1.5 \mathrm{pm} \mathrm{InGaAsPhP}$ lasers', Jpn. J. Appl. Phys., 1983, 22, pp. 1553-1557

13 Yanagawa, T., Saito, S., Machida, S., Yamamoto, Y., and Noguchi, Y. 'Frequency stabilization of 1.5-pm InGaAsP distributed feedback laser to an NH, absorption lines', Appl. Phys. Lett., 1984, 45, (8), pp. $826-828$

14 Yamaguchi, S., and Suzuki, M.: 'Frequency locking of an InGaAsP semiconductor laser to the first overtone vibration-rotation lines of hydrogen fluoride', Appl. Phys. Lett., 1982, 41, pp. 1034-1036

15 Measures, R.M.: 'Laser remote chemical analysis' (John Wiley \& Sons, 1988)

16 Henry, C.H.: 'Phase noise in semiconductor lasers', J. Lightwave Technol., 1986, LT-4, pp. 298-311

17 Henry, C.H.: 'Theory of the linewidth of semiconductor lasers', IEEE J. Quantum Electron., 1982, 18, pp. 259-264

18 Baney, D.M., and Sorin, W.V.: 'Measurement of a modulated DFB laser spectrum using gated delayed self-homodyne technique', Electron. Lett., 1988, 24, pp. 669-670

19 Derickson, D.: 'Fiber optic test and measurement' (Hewlett-Packard Professional Books (paperback)), 1998 